\title{
Laboratory Scale Photogrammetry: Micro- and Macroscopic 3D Surface Imaging
}

\author{
Daria Monaenkova, Diana Deese, Chad Schuette and Matthew Thorseth
}

Dow, Midland, Michigan, United States

All traditional photographs are merely flat projections of three dimensional objects and thus often are unable to convey the complexity of object shapes, appearances, and orientation in real space. Threedimensional models of the objects, in contrast, carry valuable information on the true object shapes, aspect ratios, and (in some cases) color gradients and textures. Industrial applications often use high-end laserbased scanning coordinate measurement machines (CMMs) to produce three dimensional models of the objects for reverse engineering, metrology, and for quality control in mass production. For less demanding applications or when the specialized equipment is unavailable, photogrammetry may become a low cost alternative to CMMs. Photogrammetry is a technique that allows for reconstruction of three-dimensional model of the object from a series of overlapping images of the object taken at multiple angles, rotations, tilts and magnifications. Photogrammetry is based on the concept that the location of each point on the object in space can be mathematically derived from planar projections of the point taken from two different viewing angles [1-2].

In this work, we will discuss an application of photogrammetry on a macro and micro scale in a laboratory setting. Our macroscale set up consists of an automated multi-axis precision rotary stage, area scan color camera and a 12' 'x12' ' light panel. The set up is typically used for sample with sizes ranging from several centimeters to tens of centimeters. The camera lens is chosen based on the object size. The camera lens is equipped with a circular polarizer to mitigate unwanted light reflections and improve the quality of the acquired images. The millimeter and smaller-size objects are imaged using a digital microscope featuring a tilting stand, built-in lighting with a polarizer option and a rotation/translation stage. In the experiments the sample is mounted on a goniometer stage to overcome limitations imposed on the imaging by the microscope tilt head geometry. In both cases images are usually collected at 10 degrees tilting and rotations steps. Two to three hundred images are collected in a typical experiment. The image collection time is defined by the level of automation of the imaging set up, ranging from a few minutes of initial setup followed by automated collections to a few hours if the images need to be collected manually.

The acquired images are imported into the open source software Meshroom for reconstruction. The software automatically identifies keypoint features on the object surface (texture details or fiducial marks) visible on multiple images and uses them to estimate the camera location relative to the object and the feature location in a three-dimensional space [1-2]. The result of this process is called a point cloud. After point cloud generation, the adjacent points can be connected to generate a basic surface mesh. However, better models are typically generated by using the point clouds to reconstruct depth maps in each image, which are then combined for the final mesh. During the last reconstruction step, the color information extracted from the images is applied to a generated surface. The reconstructed three-dimensional model of the object can be then be exported for advanced visualization, model editing, and further design.

We will summarize the general recommendations for the image acquisition on macro and micro scale that enable the best quality of the reconstruction. We will provide a comparison of the object models produced using CMM and obtained via laboratory scale photogrammetry set ups. With the increased power of modern computers and advancements in imaging technology, photogrammetry can benefit a broader audience involved in both macroscopic and microscopic imaging.

\section{References}


1. T. Luhmann, S. Robson, S. Kyle, I. Harley, Close Range Photogrammetry - Principles, techniques and applications (2011), Whittles Publishing.

2. T Luhmann, S Robson, S Kyle and I Harley Close Range Photogrammetry - Principles, techniques and applications (2006), Whittles Publishing. 